\title{
Augmentation du risque de maladies endémiques au Canada transmises par des moustiques en raison du changement climatique
}

\author{
A Ludwig ${ }^{1 *}, \mathrm{H}$ Zheng ${ }^{2}$, L Vrbova ${ }^{3}$, MA Drebot ${ }^{4}, \mathrm{M}$ Iranpour $^{4}$, LR Lindsay ${ }^{4}$
}

\section{Résumé}

Il existe actuellement plus de 80 espèces de moustiques endémiques au Canada, bien que seulement quelques-unes d'entre elles soient porteuses d'agents pathogènes pouvant provoquer des maladies chez les humains. Le virus du Nil occidental, le virus de l'encéphalite équine de l'Est et les virus du sérogroupe Californie (incluant les virus Jamestown Canyon et Snowshoe hare) sont des virus transmis par des moustiques ayant provoqué des infections chez les humains en Amérique du Nord, y compris au Canada. Au cours des 20 dernières années, l'incidence de la plupart de ces maladies endémiques transmises par des moustiques a augmenté d'environ $10 \%$ au Canada, en grande partie en raison du changement climatique. On s'attend à ce que le cycle de vie des moustiques et les modes de transmission des virus subissent les effets du changement climatique, ce qui entraînera une augmentation de l'étendue et de l'abondance locale de plusieurs espèces importantes de moustiques. Des études en laboratoire et des travaux de modélisation mathématique suggèrent que l'augmentation des températures ambiantes, les changements dans les précipitations et les phénomènes météorologiques extrêmes associés au changement climatique continueront probablement de favoriser l'expansion de la population de moustiques vecteurs et la présence de maladies transmises par des moustiques, augmentant ainsi la durée des saisons de transmission et entraînant des épidémies de ces maladies. De plus, les cycles de transmission des maladies endémiques transmises par des moustiques au Canada sont complexes, car ils englobent de multiples hôtes réservoirs (oiseaux et mammifères), de multiples agents pathogènes et de multiples espèces de moustiques sensibles au changement climatique et aux autres changements environnementaux. Par conséquent, il est difficile de prévoir les tendances émergentes possibles. Les incertitudes quant aux changements prévus mettent en évidence la nécessité de poursuivre (et d'accroître) la surveillance et la recherche afin d'assurer une évaluation opportune et exacte des risques pour la santé publique des Canadiens.
Citation proposée : Ludwig A, Zheng H, Vrbova L, Drebot MA, Iranpour M, Lindsay LR. Augmentation du risque de maladies endémiques transmises par des moustiques au Canada en raison du changement climatique. Relevé des maladies transmissibles au Canada 2019;45(4):99-107. https://doi.org/10.14745/ccdr.v45i04a03f

Mots clés : Canada, changement climatique, encéphalite équine de l'Est, endémie, maladies transmises par des moustiques, virus du Nil occidental, virus du sérogroupe Californie
Cette oeuvre est mise à la disposition selon les termes de la licence internationale Creative Commons Attribution 4.0

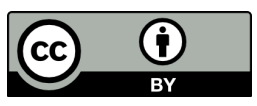

Affiliations

${ }^{1}$ Laboratoire national de microbiologie, Agence de la santé publique du Canada, SaintHyacinthe (Québec)

${ }^{2}$ Centre des maladies infectieuses d'origine alimentaire et environnementale Agence de la santé publique du Canada, Ottawa (Ontario)

\section{${ }^{3}$ Centre des maladies} infectieuses d'origine alimentaire, environnementale et zoonotique, Agence de la santé publique du Canada, Toronto (Ontario)

\author{
${ }^{4}$ Laboratoire national de \\ microbiologie, Agence de la santé \\ publique du Canada, Winnipeg \\ (Manitoba)
}

\section{${ }^{\star}$ Correspondance:}

antoinette.ludwig@canada.ca

\section{Introduction}

Le rapport du Groupe d'experts intergouvernemental des Nations Unies sur l'évolution du climat indique que les maladies transmises par des moustiques sont les maladies infectieuses les plus sensibles au changement climatique (1). Le Canada a déjà connu des changements climatiques, et les tendances observées sont notamment le réchauffement, l'augmentation des périodes de chaleur extrême et de pluies abondantes, et la diminution du nombre de jours de gel (2). Ces changements devraient s'intensifier au cours des prochaines décennies, jusqu'à ce que les émissions de gaz à effet de serre commencent à diminuer à l'échelle mondiale.
On s'attend à ce que les changements climatiques influent sur la flore et la faune du Canada de façon prévisible et imprévisible. Dans la faune susceptible d'être touchée par le changement climatique se trouvent diverses espèces de moustiques. En plus d'être considérées comme une nuisance en raison de leurs piqûres, quelques espèces peuvent également transmettre des organismes pathogènes infectieux.

Le présent document porte sur les moustiques endémiques au Canada et sur les maladies dont ils peuvent être vecteurs, soit les maladies endémiques transmises par des moustiques. 
Ces maladies se distinguent des maladies exotiques transmises par des moustiques qu'on peut contracter à l'extérieur du Canada, quoique ces dernières pourraient apparaître au Canada dans I'avenir (3). L'infection par le virus du Nil occidental est la maladie endémique transmise par des moustiques la plus importante au Canada sur le plan médical. Les autres maladies endémiques transmises par des moustiques comprennent le virus de l'encéphalite équine de l'Est (virus EEE) et deux virus du sérogroupe Californie : le virus Jamestown Canyon et le virus Snowshoe hare (4-8). Toutes ces maladies sont transmises par des arbovirus endémiques (acronyme d'ARthropod-BOrne virus, qui désigne tout virus transmis par un vecteur arthropode). Les arthropodes comprennent les moustiques, les tiques et les mouches noires. Le présent document porte seulement sur les moustiques. Les arthropodes vecteurs sont des espèces à sang froid et sont donc particulièrement sensibles aux facteurs climatiques.

Cette étude vise à donner une vue d'ensemble des moustiques observés au Canada, à résumer comment le changement climatique peut accroître le risque de maladies endémiques transmises par des moustiques, à discuter de telles maladies causées par des moustiques susceptibles de se multiplier en milieux urbain et rural et à déterminer ce qui peut être fait pour réduire ces risques.

\section{Espèces de moustiques endémiques au Canada}

Les quatre principaux arbovirus endémiques au Canada (le virus du Nil occidental, le virus EEE, le virus Jamestown Canyon et le virus Snowshoe hare) sont tous transmis par des piqûres de moustiques femelles infectés par des agents pathogènes contractés d'hôtes réservoirs mammifères ou aviaires bien précis (9). Les principaux moustiques vecteurs du virus du Nil occidental sont le Culex pipiens et le Cx.restuans dans l'Est du Canada et le Cx.tarsalis dans l'Ouest du Canada (10). Le principal vecteur du virus EEE est le Culiseta melanura $(11,12)$ et les principaux vecteurs des virus du sérogroupe Californie sont une variété d'espèces de moustiques de catégories autres que le Culex (p.ex., les espèces Aedes, Culiseta et Anopheles) (13-15). Les virus Jamestown Canyon et Snowshoe hare font partie du sérogroupe Californie. Les réservoirs de ces pathogènes varient selon les espèces de moustiques; par exemple, le principal réservoir animal du virus Jamestown Canyon est le cerf de Virginie (16), tandis que celui du virus Snowshoe hare est (sans surprise) le lièvre d'Amérique et d'autres petits mammifères (8). Les humains sont des hôtes accidentels ou «terminaux» des arbovirus endémiques, ce qui signifie que bien qu'ils puissent être infectés, ils ne peuvent pas transmettre ces virus efficacement aux moustiques qui se nourrissent de leur sang en raison d'une virémie faible et transitoire $(4,10)$. Les arbovirus peuvent aussi parfois être transmis à la suite d'une transfusion sanguine ou d'une greffe de tissus $(17,18)$.
En plus de ces moustiques traditionnellement endémiques, de nombreuses autres espèces ont été introduites au Canada au cours des dernières décennies. Dans les années 1970, un examen exhaustif des insectes et des arachnides du Canada a révélé qu'il y avait 74 espèces de moustiques (19). Au cours des 40 années qui ont suivi, six espèces ont été déclarées comme nouvellement établies au Canada : Ochlerotatus ventrovittis; Oc. japonicus; Cx. salinarius; Cx. erraticus; An. perplexens; et An. crucians [(20), et données non publiées de $M$. Iranpour]. De plus, dix espèces ont étendu leur territoire au Canada : Uranotaenia sapphirina; Cs. melanura; Cs. minnesotae; Cx. tarsalis; Oc. sticticus; Oc. spencerii; Oc. dorsalis; Oc. nigromaculis; Oc. campestris; et Oc. cataphylla (21). Ainsi, on trouve maintenant environ 80 espèces de moustiques au Canada.

Récemment, des espèces envahissantes du type Aedes ont été observées dans le sud de l'Ontario et du Québec. L'espèce Aedes albopictus a été détectée en faible nombre dans certaines parties du comté de Windsor-Essex, dans le sud de l'Ontario, en 2016, 2017 et 2018 (22). La détection répétée de cette espèce sur un certain nombre de sites de collecte suggère que cette espèce est en voie de devenir endémique dans cette partie du Canada. Des spécimens d'Ae. aegypti ont également été prélevés sur certains de ces mêmes sites à Windsor en Ontario, en 2016 et 2017, et sur un seul site dans le sud du Québec, en 2017. Bien que ces deux souches d'Aedes soient bien reconnues pour transmettre des maladies exotiques dont les agents sont des moustiques non indigènes du Canada (3), elles peuvent aussi être un vecteur de maladies transmises par des moustiques déjà endémiques au Canada, dont le virus du Nil occidental.

\section{Le changement climatique accroîtra les risques de maladies endémiques transmises par des moustiques}

Les principaux aspects du changement climatique qui ont des effets sur les moustiques endémiques sont les hausses de température et les fluctuations des précipitations. Des précipitations plus abondantes augmentent généralement l'étendue potentielle des sites de ponte et des gîtes larvaires des moustiques dans l'environnement. Leur lien est souvent non linéaire. Les précipitations supérieures à la moyenne rendent généralement les moustiques plus abondants en leur donnant accès à plus d'eau stagnante, tandis que des précipitations excessives ou violentes peuvent lessiver et détruire les œufs, et chasser les larves vivant dans des habitats sélectionnés (23). Des températures élevées peuvent accélérer le développement des moustiques aux stades du cycle de vie où ils n'ont pas atteint la maturité, et ainsi, mener à des taux de reproduction plus élevés et à une augmentation exponentielle de leur population $(24,25)$. Ces températures élevées réduisent la période d'incubation extrinsèque, de sorte que les moustiques qui ont contracté l'infection deviennent infectieux plus tôt; par exemple, les 
éclosions d'infection par le virus du Nil occidental semblent se produire plus fréquemment au Canada lorsque les températures saisonnières sont supérieures à la moyenne, car ces conditions favorisent l'acquisition rapide du virus chez les moustiques vecteurs et prolongent la recherche d'hôtes par les moustiques femelles potentiellement infectés (26). II a été signalé qu'en Corée et au Japon, la durée de la saison de transmission peut être prolongée de plusieurs mois lorsque les températures moyennes estivales augmentent d'aussi peu que de $5^{\circ} \mathrm{C}$ (27). Des changements dans les précipitations augmentent la disponibilité d'eau stagnante, où les moustiques pondent leurs œufs et où vivent les moustiques qui n'ont pas atteint la maturité. Par conséquent, ces changements ont une forte incidence sur la reproduction des moustiques $(28,29)$.

L'incidence du changement climatique sur la transmission du virus du Nil occidental au Canada a fait l'objet de deux études, dont les conclusions sont similaires $(30,31)$. Chen et ses collaborateurs (30) ont examiné la transmission du virus du Nil occidental dans les Prairies (où le Cx. tarsalis est le principal vecteur) et ont prévu une prolongation de l'activité saisonnière du Cx. tarsalis infecté par le virus du Nil occidental allant de trois mois (de juin à août) à cinq mois (de mai à septembre), d'ici les années 2080. Ces auteurs ont également prédit que le Cx. tarsalis et le virus du Nil occidental s'étendraient au nord. Hongoh et ses collaborateurs (31) ont modélisé la répartition potentielle des populations de Cx. pipiens dans l'Est du Canada et prédit une expansion similaire au nord pour ce vecteur du virus du Nil occidental.

Les oiseaux et certains mammifères constituent d'importants réservoirs pour le virus du Nil occidental et d'autres maladies transmises par des moustiques. Dans le cas du virus du Nil occidental, un vaste éventail d'espèces d'oiseaux sert d'hôtes, dont les corvidés (corneilles, geais et pies) et les passereaux (rouges-gorges, moineaux, pinsons et étourneaux) $(10,32$ 34). Pour ce qui est des autres maladies transmises par des moustiques, des mammifères tels que les cerfs, les écureuils, les suisses et les lièvres, sont les hôtes réservoirs. Le changement climatique pourrait agir de plusieurs façons sur ces populations d'hôtes réservoirs, par exemple sur leur abondance et la répartition des espèces. II est possible que le changement climatique puisse également avoir une incidence sur la santé individuelle et globale des hôtes réservoirs, car les virus les plus abondants pourraient aussi leur nuire directement.

Il est important de noter que la prolifération des moustiques et des maladies qu'ils transmettent peut également dépendre d'autres changements environnementaux, y compris les changements dans l'exploitation des terres, ainsi que les activités de lutte antivectorielle $(35,36)$. La perte et la fragmentation de I'habitat (37) peuvent également avoir des conséquences sur les réservoirs des oiseaux et des mammifères (38).

\section{Maladies endémiques actuelles et émergentes transmises par des moustiques}

Les diverses espèces de moustiques ont des caractéristiques différentes en ce qui concerne leurs habitats de prédilection et leur charge pathogène. En raison du changement climatique, la prévalence du virus du Nil occidental pourrait augmenter dans les zones urbaines et rurales, tandis que d'autres maladies transmises par des moustiques, comme le virus EEE et les virus du sérogroupe Californie, pourraient gagner en importance, particulièrement dans les zones rurales. Voici une brève description des maladies endémiques actuelles et émergentes transmises par des moustiques endémiques.

\section{Le virus du Nil occidental pourrait être à la hausse dans les régions rurales et urbaines} Le virus du Nil occidental est transmis par le $C x$. pipiens, un moustique surtout urbain, et le $C x$. tarsalis, un moustique surtout rural. Le changement climatique peut influer sur les risques associés au virus du Nil occidental, en particulier dans les régions urbaines de l'Est et dans les régions rurales des Prairies. L'augmentation du nombre de moustiques, y compris ceux qui sont infectés par des maladies transmises par des moustiques, aura des répercussions plus importantes sur la santé publique dans les zones urbaines, car c'est là que réside la grande majorité des Canadiens. Les premiers symptômes du virus du Nil occidental sont notamment de la fièvre, des maux de tête, des éruptions cutanées, des nausées et des douleurs musculaires. La plupart des personnes touchées se rétablissent complètement, mais environ $1 \%$ d'entre elles développent une maladie grave (méningite, encéphalite, paralysie flasque aiguë et poliomyélite). Les personnes âgées de plus de 70 ans qui souffrent de pathologies sous-jacentes et celles qui sont immunodéprimées courent un plus grand risque de contracter une maladie grave (39-42).

Depuis 2002, le nombre de cas déclarés de virus du Nil occidental chez l'humain a fluctué considérablement, passant de 1481 cas en 2003 à cinq cas en 2010 et à 2215 cas en 2007, ce qui peut être dû en partie aux variations météorologiques ayant une incidence sur la reproduction des moustiques et la transmission du virus, ainsi qu'aux variations dans la déclaration des manifestations (43). La variation géographique du nombre de cas déclarés de virus du Nil occidental chez l'humain a également été spectaculaire : en 2003 et 2007, la plupart des cas de virus du Nil occidental chez l'humain ont été déclarés dans les régions des Prairies (Alberta, Saskatchewan et Manitoba), mais en 2002, 2012 et 2018, la plupart des cas sont survenus en Ontario et au Québec (43). Cette variation géographique est probablement associée aux effets locaux des conditions météorologiques sur les différents vecteurs et sur la transmission du virus, ainsi qu'à une certaine variation dans la déclaration des manifestations $(28,29,44)$. 


\section{Augmentation prévue des cas d'encéphalite équine de l'Est et de virus du sérogroupe Californie dans les zones rurales}

Le virus de l'encéphalite équine de l'Est est transmis par les moustiques de type $C$. melanura qui se reproduisent dans les marécages d'eau douce $(11,12)$. Le réservoir de ce virus est constitué d'hôtes aviaires. L'encéphalite équine de l'Est peut être asymptomatique ou se présenter sous deux formes : systémique ou encéphalitique. Environ le tiers des personnes atteintes d'encéphalite en mourront (45). Un seul cas de virus EEE humain a été déclaré en Ontario en 2016 (43). L'encéphalite équine de I'Est a connu des éclosions sporadiques chez les chevaux (et les oiseaux exotiques) en Ontario depuis 1939 (46). Des éclosions atypiques importantes du virus EEE chez les chevaux ont été déclarées en Ontario, au Québec et en Nouvelle-Écosse en 2008, 2009 et 2010 (46).

Les virus du sérogroupe Californie sont transmis par un certain nombre d'espèces de moustiques. Ils peuvent provoquer des maladies fébriles et neurologiques (47). Après la mise en œuvre de nouvelles méthodes d'analyse en 2005, plus de 200 cas probables et confirmés de virus du sérogroupe Californie ont été déclarés à l'Agence de la santé publique du Canada ou à des laboratoires provinciaux de santé publique entre 2005 et 2014 (données non publiées de M. Drebot). Des cas ont été recensés dans toutes les provinces ainsi que dans les Territoires du Nord-Ouest et dans d'autres régions du Nord. De plus, des études de séroprévalence ont révélé des taux d'exposition allant de 20 à $40 \%$ ou plus dans certaines régions du Canada. [(48-50) et données non publiées de M. Drebot]. Depuis 2015, de 20 à 40 cas d'infection chez l'humain ont été observés chaque année au Canada, à l'exception de 2017, alors que 122 cas avaient été déclarés (43). Cette augmentation spectaculaire peut être attribuable, du moins en partie, à l'amélioration du dépistage chez les personnes atteintes d'une maladie semblable à celle du virus du Nil occidental (les tests de dépistage du virus du Nil occidental étaient négatifs, de sorte que d'autres tests ont été demandés). D'après le Rapport de surveillance du virus du Nil occidental et autres arbovirus, il y a eu plus de 100 cas de virus Jamestown Canyon et Snowshoe hare au Québec en 2017 (H. Zheng, H. Wood et M. Drebot, Rapport de surveillance du virus du Nil occidental et autres arbovirus transmis par les moustiques au Québec, rapport non publié). Il est fort probable que les cas de virus du sérogroupe Californie soient sous-diagnostiqués en raison du faible niveau de sensibilisation à ces pathogènes chez les médecins et les autres professionnels de la santé.

\section{Intervention clinique et de santé publique actuelle}

En l'absence de vaccins ou de traitements particuliers, la réduction de l'habitat des moustiques, la réduction des piqûres de moustiques, les tests précoces et précis de détection des maladies transmises par des moustiques chez les humains et la surveillance continue sont au cœur de l'intervention clinique et de santé publique.

\section{Mesures préventives: prévenir les infections en évitant les piqûres de moustiques}

Il n'existe pas de vaccin ni de traitement particulier contre le virus du Nil occidental, le virus EEE ou les virus du sérogroupe Californie, de sorte que la prévention des piqûres de moustiques infectieux est la clé pour les maîtriser (43). On peut réduire les piqûres en se couvrant la peau (par exemple, en portant des pantalons longs et des chemises amples à manches longues) ou en utilisant un insectifuge contenant les produits chimiques $\mathrm{N}, \mathrm{N}$-diéthyl-3-méthylbenzamide (DEET) ou icaridine (51). Il est également important de réduire la superficie de l'habitat des moustiques près des habitations, principalement en éliminant l'eau stagnante. Les municipalités, souvent en collaboration avec les gouvernements provinciaux, ont financé des programmes de réduction des moustiques conçus pour diminuer la taille des populations de moustiques nuisibles et vecteurs. La portée de ces programmes varie énormément à travers le Canada, mais elle comprend habituellement la réduction à la source (élimination de l'eau stagnante), l'application de larvicides dans l'eau stagnante et, moins fréquemment, des traitements de pulvérisation aérienne ou de végétaux contenant des produits conçus pour tuer les moustiques adultes.

\section{Diagnostic précoce et dépistage des arbovirus}

La détection précoce des maladies transmises par des moustiques est importante pour éviter des complications potentiellement graves. Le diagnostic des infections par les arbovirus peut comporter des difficultés, car initialement, les patients présentent souvent des symptômes non spécifiques. Le diagnostic des maladies transmises par des moustiques doit être confirmé par des épreuves de laboratoire. Le Laboratoire national de microbiologie (Winnipeg) est le laboratoire de référence pour les tests de dépistage des virus EEE et du sérogroupe Californie et pour le dépistage du virus du Nil occidental dans les provinces où sa prévalence est faible (Terre-Neuve-et-Labrador, Île-du-Prince-Édouard, Nouvelle-Écosse et Nouveau-Brunswick). Les provinces où le nombre de cas de virus du Nil occidental est le plus élevé effectuent leurs propres tests.

\section{Surveillance}

L'Organisation mondiale de la Santé a souligné l'importance d'identifier et de surveiller diverses populations de vecteurs dans le cadre de la surveillance mondiale, y compris les moustiques qui peuvent transporter et transmettre des arbovirus, car il est plus opportun de surveiller les risques chez les populations de moustiques que d'attendre l'apparition de cas chez l'humain (52).

À l'heure actuelle, la surveillance des arbovirus varie quelque peu d'une province ou d'un territoire à l'autre au Canada et les efforts ont été concentrés en grande partie dans les Prairies, en Ontario et au Québec, où l'incidence de la maladie est 
généralement plus élevée. Dans le cas du virus du Nil occidental, la surveillance des moustiques consiste à compter les différentes espèces de moustiques et à calculer le taux d'infectiosité des espèces porteuses du virus du Nil occidental. Cette approche de surveillance doit s'étendre à d'autres zones géographiques et à d'autres maladies transmises par des moustiques qui font l'objet d'une surveillance. Ce faisant, il est possible de suivre l'évolution des frontières du virus du Nil occidental et de détecter l'émergence de moustiques infectés par le virus EEE, le virus Jamestown Canyon, le virus Snowshoe hare et d'autres virus, afin d'éclairer les décisions sur les mesures de santé publique.

Étant donné que le virus du Nil occidental est actuellement une maladie à déclaration obligatoire au Canada, la surveillance comprend l'incidence des infections chez l'humain, y compris la détection de la présence du virus du Nil occidental dans les dons de sang (tous les dons sont soumis à des tests de dépistage d'un large éventail de maladies et de pathogènes). De plus, les tendances en matière de mortalité et de morbidité chez les populations d'oiseaux sauvages, les chevaux et d'autres animaux domestiques font l'objet d'une surveillance. Ces données sont recueillies par les provinces et les territoires aux fins d'intervention par ces administrations, mais l'information recueillie dans le cadre de la surveillance est également transmise à l'Agence de la santé publique du Canada, qui en fait la synthèse et l'analyse afin de dresser un tableau national et de le transmettre aux partenaires provinciaux et territoriaux chaque les semaines (43).

Étant donné que les infections par les virus EEE, Jamestown Canyon et du sérogroupe Californie ne sont pas actuellement des maladies à déclaration obligatoire partout au Canada, il n'existe aucun programme national de surveillance de leur présence chez les moustiques, les réservoirs ou les humains. Néanmoins, le Laboratoire national de microbiologie a effectué des tests de dépistage de ces arbovirus chez des patients qui présentaient des symptômes compatibles avec des infections à arbovirus, ce qui a permis de détecter des cas d'infection par le virus Jamestown Canyon et le virus Snowshoe hare $(8,43)$. II a également effectué des études de séroprévalence pour d'autres virus de type Orthobunyavirus, comme le virus de Cache Valley. Le virus de Cache Valley peut causer des maladies neurologiques chez l'humain et le bétail (53), et des cas de maladie symptomatique ont été associés à des sérologies positives chez des patients du Manitoba, de la Saskatchewan et de l'Alberta (données non publiées de M. Drebot).

\section{Discussion}

Le changement climatique influera sans aucun doute sur la propagation des virus par des moustiques endémiques dans l'avenir, en raison de l'augmentation du nombre et des types d'espèces endémiques (y compris celles qui sont associées aux arbovirus) et l'apparition de nouvelles espèces (et de pathogènes associés) au Canada. II est difficile de prévoir la façon dont les maladies transmises par des moustiques réagiront au changement climatique : les moustiques, les réservoirs et l'environnement réagissent différemment au changement climatique $(54,55)$. Cela signifie que même un changement climatique modéré peut entraîner une forte augmentation de la transmission des arbovirus $(1,56)$.

De plus, chaque maladie transmise par des moustiques possède des cycles de transmission, des réservoirs et des vecteurs uniques. Ces derniers peuvent $n$ 'exister que dans certaines régions du Canada, de sorte que les changements dans la prévalence des maladies transmises par des moustiques seront différents d'une région, d'un paysage ou d'un habitat à l'autre. On s'attend à ce que la prévalence du virus du Nil occidental augmente dans les régions rurales et urbaines et que celle des autres arbovirus actuellement endémiques (c.-à-d. les virus EEE et du sérogroupe Californie) augmente dans les régions rurales.

Il est nécessaire d'examiner plus à fond les répercussions possibles du changement climatique sur le virus du Nil occidental, le virus EEE et les virus du sérogroupe Californie au Canada. Idéalement, ces études devraient considérer et modéliser chaque cycle de transmission dans son ensemble, et non se concentrer sur un seul aspect, par exemple, la densité de moustiques ou la dynamique des populations de réservoirs. Il sera important d'accroître notre capacité de surveillance, non seulement de l'expansion des arbovirus existants dans de nouvelles zones géographiques, mais aussi de l'apparition de nouveaux arbovirus, et de mener des recherches pour mieux comprendre la dynamique changeante et potentiellement croissante de la transmission des arbovirus.

Le portrait des espèces de moustiques endémiques au Canada est en constante évolution, et ces changements dans les espèces de moustiques (type, répartition et activité) sont exacerbés par le changement climatique; les cliniciens et les professionnels de la santé publique devront prendre conscience des répercussions que ces changements peuvent avoir sur les maladies transmises par des moustiques au Canada. Nous prévoyons que le nombre moyen de cas augmentera, parallèlement à une augmentation du nombre de types d'arbovirus endémiques, d'une manière relativement sporadique et imprévisible. Les professionnels de la santé publique de première ligne ainsi que les laboratoires d'épreuves et de surveillance devront faire preuve d'une vigilance accrue.

\section{Conclusion}

On s'attend à ce que le changement climatique ait des effets importants sur les populations endémiques de moustiques au Canada et, de ce fait, sur les maladies transmises par des moustiques, comme le virus du Nil occidental, le virus EEE et les virus du sérogroupe Californie. Les maladies transmises par des moustiques possèdent des cycles de transmission complexes, englobant de multiples hôtes réservoirs (oiseaux et mammifères), et parfois, de multiples espèces de moustiques, pouvant tous être sensibles de façon différente aux changements climatique 
et environnementaux. Comme le virus du Nil occidental est une maladie à déclaration obligatoire, il existe des données sur la prévalence de la maladie et sur son évolution au fil du temps, mais on en sait beaucoup moins sur le virus EEE et les virus du sérogroupe Californie. Leur déclaration et leur traitement sont complexes, car les symptômes des maladies transmises par des moustiques peuvent être assez imprécis. L'apparition sporadique de certaines maladies transmises par des moustiques a entravé la mise en œuvre de tests diagnostiques pour certains arbovirus à l'échelle provinciale. Ainsi, la centralisation des tests (au Laboratoire national de microbiologie) est plus rentable pour certains arbovirus.

Les changements prévus chez les moustiques et dans les maladies transmises par des moustiques causés par le changement climatique font ressortir la nécessité de poursuivre la surveillance et la recherche afin d'assurer une évaluation opportune et exacte des risques pour la santé publique des Canadiens. Les professionnels de la santé publique et les cliniciens doivent sensibiliser la population canadienne à cet important risque pour la santé publique et faire preuve de vigilance quant à l'émergence et à la propagation de nouvelles souches de moustiques et de nouvelles maladies transmises par des moustiques.

\section{Déclaration des auteurs}

$\mathrm{AL}$ - Auteur principal

$\mathrm{HZ}$ - Corédacteur et réviseur des ébauches

LV - Corédacteur et réviseur des ébauches MAD - Corédacteur et réviseur des ébauches $\mathrm{LRL}$ - Corédacteur et réviseur des ébauches $\mathrm{MI}$ - Corédacteur et réviseur des ébauches

\section{Conflit d'intérêts}

Aucun.

\section{Financement}

Ce travail a été réalisé grâce au soutien de l'Agence de la santé publique du Canada.
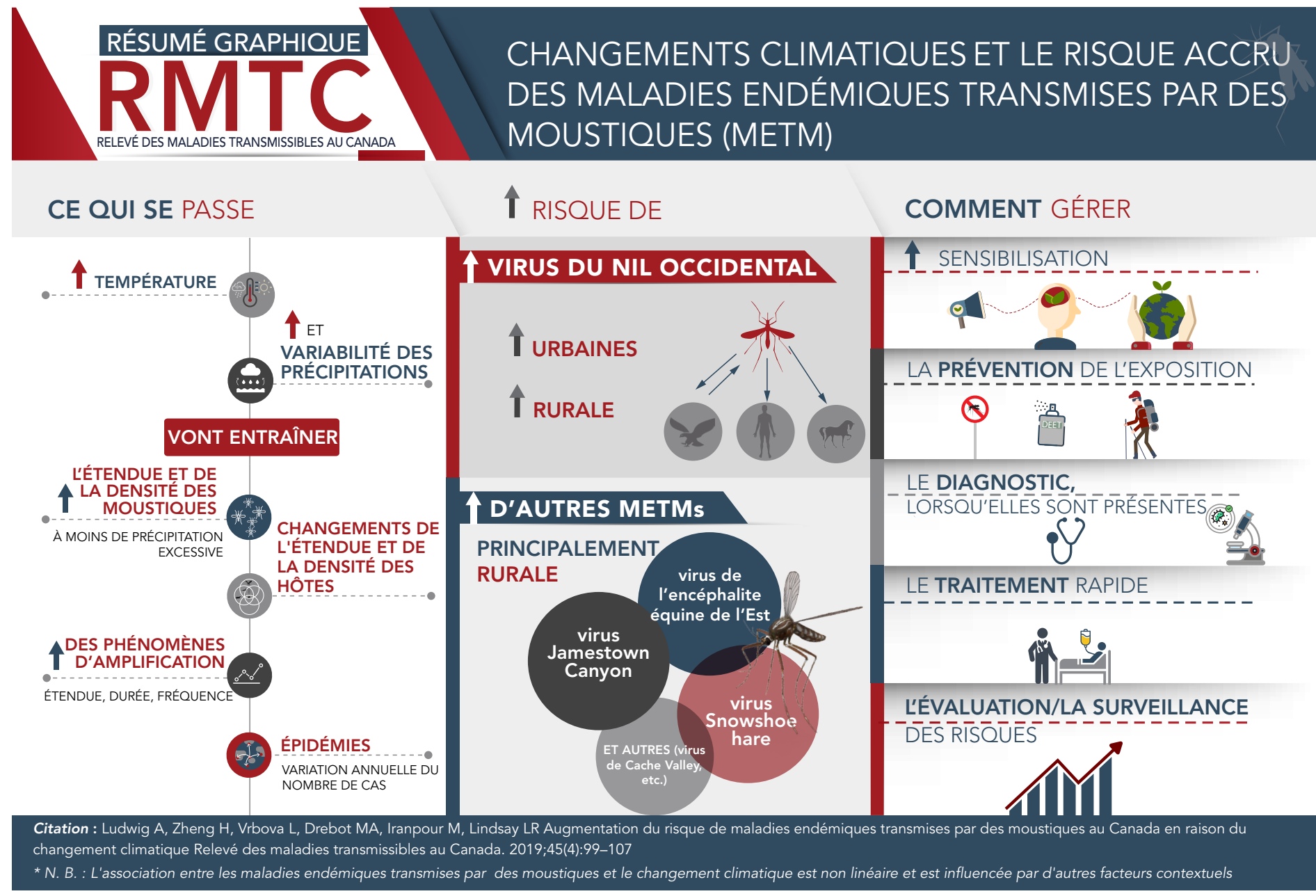


\section{Références}

1. Smith KR, Woodward A, Campbell-Lendrum D, Chadee DD, Honda Y, Liu Q, Olwoch JM, Revich B, Sauerborn R. Climate Change 2014: Impacts, Adaptation, and Vulnerability. Part A: Global and Sectoral Aspects. Contribution of Working Group II to the Fifth Assessment Report of the Intergovernmental Panel on Climate Change (WG11AR5). Cambridge (UK) and New York (NY): Cambridge University Press; 2014. Chapter 11, Human health: impacts, adaptation, and co-benefits; pp. 709-54. www.ipcc.ch/site/assets/uploads/2018/02/WGIIAR5Chap11_FINAL.pdf

2. Romero-Lankao P, Smith JB, Davidson DJ, Diffenbaugh NS, Kinney PL, Kirshen P, Kovacs P, Villers Ruiz L. Climate Change 2014: Impacts, Adaptation, and Vulnerability. Part B: Regional Aspects. Contribution of Working Group II to the Fifth Assessment Report of the Intergovernmental Panel on Climate Change (WG11AR5). Cambridge (UK) and New York (NY): Cambridge University Press; 2014. Chapter 26, North America; pp. 1439-98. https://www.ipcc.ch/report/ar5/wg2/

3. $\mathrm{Ng} \mathrm{V}$, Rees EE, Lindsay LR, Drebot MA, Brownstone T, Sadeghieh T, Khan SU. Les changements climatiques pourraient-ils entraîner la propagation de maladies exotiques transmises par les moustiques au Canada? Relevé des maladies transmissibles au Canada 2019;45(4):108-18. DOI

4. Kulkarni MA, Berrang-Ford L, Buck PA, Drebot MA, Lindsay $\mathrm{LR}$, Ogden NH. Major emerging vector-borne zoonotic diseases of public health importance in Canada. Emerg Microbes Infect 2015 Jun;4(6):e33. PubMed

5. Rocheleau JP, Michel P, Lindsay LR, Drebot M, Dibernardo A, Ogden NH, Fortin A, Arsenault J. Risk factors associated with seropositivity to California serogroup viruses in humans and pet dogs, Quebec, Canada. Epidemiol Infect 2018 Jul;146(9):1167-76. DOl PubMed

6. Zheng H, Drebot MA, Coulthart MB. Le virus du Nil occidental au Canada : un virus en évolution permanente, mais présent pour de bon. Relevé des maladies transmissibles au Canada 2014;40(10):199-204. DOI

7. Patriquin $G$, Drebot $M$, Cole $T$, Lindsay $R$, Schleihauf $E$, Johnston BL, Dimitrova K, Traykova-Andonova M, Mask A, Haldane D, Hatchette TF High seroprevalence of Jamestown Canyon virus among deer and humans, Nova Scotia, Canada. Emerg Infect Dis 2018 Jan;24(1):118-21. DOI PubMed

8. Drebot MA. Bunyavirus transmis par les moustiques émergents au Canada. Relevé des maladies transmissibles au Canada 2015;41(6):133-41. DOI

9. Grubaugh ND, Ebel GD. Dynamics of West Nile virus evolution in mosquito vectors. Curr Opin Virol 2016 Dec;21:132-8. DOl PubMed

10. Kramer LD, Styer LM, Ebel GD. A global perspective on the epidemiology of West Nile virus. Annu Rev Entomol 2008;53:61-81. DOl PubMed

11. Armstrong PM, Andreadis TG. Eastern equine encephalitis virus in mosquitoes and their role as bridge vectors. Emerg Infect Dis 2010 Dec;16(12):1869-74. DOl PubMed
12. Molaei G, Andreadis TG, Armstrong PM, Anderson JF, Vossbrinck CR. Host feeding patterns of Culex mosquitoes and West Nile virus transmission, northeastern United States. Emerg Infect Dis 2006 Mar;12(3):468-74. DOI PubMed

13. LeDuc JW. Epidemiology and ecology of the California serogroup viruses. Am J Trop Med Hyg 1987 Nov;37(3 Suppl):60S-8S. DOI PubMed

14. Pastula DM, Hoang Johnson DK, White JL, Dupuis AP 2nd, Fischer M, Staples JE. Jamestown Canyon Virus Disease in the United States-2000-2013. Am J Trop Med Hyg 2015 Aug;93(2):384-9. DOI PubMed

15. Webster D, Dimitrova K, Holloway K, Makowski K, Safronetz D, Drebot MA. California Serogroup Virus Infection Associated with Encephalitis and Cognitive Decline, Canada, 2015. Emerg Infect Dis 2017 Aug;23(8):1423-4. DOl PubMed

16. Andreadis TG, Anderson JF, Armstrong PM, Main AJ. Isolations of Jamestown Canyon virus (Bunyaviridae: Orthobunyavirus) from field-collected mosquitoes (Diptera: Culicidae) in Connecticut, USA: a ten-year analysis, 19972006. Vector Borne Zoonotic Dis 2008 Apr;8(2):175-88. DOI PubMed

17. Agence de la santé publique du Canada. Fiche technique santé-sécurité: agents pathogènes - Virus du Nil occidental (VNO). Ottawa (ON): ASPC 2018. www.phac-aspc.gc.ca/lab$\mathrm{bio/res/psds-ftss/wnv-vno-fra.php}$

18. Fonseca K, Prince GD, Bratvold J, Fox JD, Pybus $M$, Preksaitis JK, Tilley P. West Nile virus infection and conjunctival exposure. Emerg Infect Dis 2005 Oct;11(10):1648-9. DOI PubMed

19. Wood DM, Dang PT, Ellis RA. The Insects and Arachnids of Canada. Part 6: The Mosquitoes of Canada Diptera: Culicidae. Publication 1686. Ottawa (ON) : Biosystematics Research Institute, Research Branch, Agriculture Canada; 2012. https://issuu.com/agropec/docs/insects_and_ arachnids_part_6

20. Giordano BV, Gasparotto A, Hunter FF. A checklist of the 67 mosquito species of Ontario, Canada. J Am Mosq Control Assoc 2015 Mar;31(1):101-3. DOl PubMed

21. Iranpour $M$, Lindsay LR, Dibernardo A. Culiseta melanura (Diptera: Culicidae), a new record for the Manitoba mosquito fauna. Proc Entomol Soc Manitoba. 2009;5:21-5. https:// home.cc.umanitoba.ca/ fieldspg/pdf/Iranpour_et_al_2009. pdf

22. June CB. 5, 2018. www.cbc.ca/news/canada/windsor/ mosquito-trap-captures-known-carriers-zika-virus-1.4693081

23. Clements AN. The Biology of Mosquitoes, Vol 1, Development, Nutrition and Reproduction. CABI Publishing; 1992. 532 p. www.cabi.org/bookshop/book/9780851993744

24. Alto BW, Juliano SA. Temperature effects on the dynamics of Aedes albopictus (Diptera: Culicidae) populations in 
the laboratory. J Med Entomol 2001 Jul;38(4):548-56. DOI PubMed

25. Semenza JC, Suk JE, Estevez V, Ebi KL, Lindgren E. Mapping climate change vulnerabilities to infectious diseases in Europe. Environ Health Perspect 2012 Mar;120(3):385-92. DOI PubMed

26. Mallya S, Sander B, Roy-Gagnon MH, Taljaard M, Jolly A, Kulkarni MA. Factors associated with human West Nile virus infection in Ontario: a generalized linear mixed modelling approach. BMC Infect Dis 2018 Mar;18(1):141. DOI PubMed

27. Lee SH, Nam KW, Jeong JY, Yoo SJ, Koh YS, Lee S, Heo ST, Seong SY, Lee KH. The effects of climate change and globalization on mosquito vectors: evidence from Jeju Island, South Korea on the potential for Asian tiger mosquito (Aedes albopictus) influxes and survival from Vietnam rather than Japan. PLoS One 2013 Jul;8(7):e68512. DOI PubMed

28. Wang J, Ogden NH, Zhu H. The impact of weather conditions on Culex pipiens and Culex restuans (Diptera: Culicidae) abundance: a case study in Peel Region. J Med Entomol 2011 Mar;48(2):468-75. DOI

29. Yoo EH, Chen D, Diao C, Russell C. The Effects of Weather and Environmental Factors on West Nile Virus Mosquito Abundance in Greater Toronto Area. Earth Interact 2016;20(3):1-22.DOI

30. Chen CC, Jenkins E, Epp T, Waldner C, Curry PS, Soos C Climate change and West Nile virus in a highly endemic region of North America. Int J Environ Res Public Health 2013 Jul;10(7):3052-71. DOl PubMed

31. Hongoh V, Berrang-Ford L, Scott ME, Lindsay LR. Expanding geographical distribution of the mosquito, Culex pipiens, in Canada under climate change. Appl Geogr 2012;33(1):5362. DOI

32. Reisen WK. Ecology of West Nile virus in North America. Viruses 2013 Sep;5(9):2079-105. DOl PubMed

33. Ludwig A, Bigras-Poulin M, Michel P, Bélanger D. Risk factors associated with West Nile virus mortality in American Crow populations in Southern Quebec. J Wildl Dis 2010 Jan;46(1):195-208. DOI

34. Kilpatrick AM, Kramer LD, Jones MJ, Marra PP, Daszak P. West Nile virus epidemics in North America are driven by shifts in mosquito feeding behavior. PLoS Biol 2006 Apr;4(4):e82. DOI PubMed

35. Lafferty KD. The ecology of climate change and infectious diseases. Ecology 2009 Apr;90(4):888-900. DOI PubMed

36. Wilson K. Climate change and the spread of infectious ideas. Ecology 2009 Apr;90(4):901-2. DOI PubMed

37. Ressources naturelles Canada. Vivre avec les changements climatiques au Canada : perspectives des secteurs relatives aux impacts et à l'adaptation. Warren FJ, Lemmen DS, editors. Ottawa (ON) : Gouvernement du Canada;
2014. 286 p. https://www.rncan.gc.ca/environnement/ ressources/publications/impacts-adaptation/rapports/ evaluations/2014/16310

38. Berteaux D, Stenseth NC. Measuring, understanding and projecting the effects of large-scale climatic variability on mammals. Clim Res 2006;32(2):95-7.

39. Petersen LR, Carson PJ, Biggerstaff BJ, Custer B, Borchardt SM, Busch MP. Estimated cumulative incidence of West Nile virus infection in US adults, 1999-2010. Epidemiol Infect 2013 Mar;141(3):591-5. DOI PubMed

40. Petersen LR, Brault AC, Nasci RS. West Nile virus: review of the literature. JAMA 2013 Jul;310(3):308-15. DOI PubMed

41. Sejvar JJ. Clinical manifestations and outcomes of West Nile virus infection. Viruses 2014 Feb;6(2):606-23. DOI PubMed

42. Badawi A, Velummailum R, Ryoo SG, Senthinathan A, Yaghoubi S, Vasileva D, Ostermeier E, Plishka M, Soosaipillai $\mathrm{M}$, Arora P. Prevalence of chronic comorbidities in dengue fever and West Nile virus: A systematic review and meta-analysis. PLoS One 2018 Jul;13(7):e0200200. DOI PubMed

43. Agence de la santé publique du Canada. Surveillance du virus du Nil occidental. Ottawa (ON) : ASPC; 2018 https:// www.canada.ca/fr/sante-publique/services/maladies/virus-niloccidental/surveillance-virus-nil-occidental.html

44. Ripoche M, Campagna C, Ludwig A, Ogden NH, Leighton PA. Short-term Forecasting of Daily Abundance of West Nile Virus Vectors Culex pipiens-restuans (Diptera: Culicidae) and Aedes vexans Based on Weather Conditions in Southern Québec (Canada). J Med Entomol 2019 Feb; DOI PubMed

45. Centers for Disease Control and Prevention. Eastern Equine Encephalitis. Symptoms and Treatment. www.cdc.gov/ easternequineencephalitis/tech/symptoms.html

46. Chénier S, Côté G, Vanderstock J, Macieira S, Laperle A, Hélie P. An eastern equine encephalomyelitis (EEE) outbreak in Quebec in the fall of 2008. Can Vet J 2010 Sep;51(9):1011-5. PubMed

47. Webster D, Dimitrova K, Holloway K, Makowski K, Safronetz $D$, Drebot MA. California serogroup virus infection associated with encephalitis and cognitive decline, Canada, 2015. Emerg Infect Dis 2017 Aug;23(8):1423-4. DOI PubMed

48. Patriquin $\mathrm{G}$, Drebot $\mathrm{M}$, Cole $\mathrm{T}$, Lindsay $\mathrm{R}$, Schleihauf $\mathrm{E}$, Johnston BL, Dimitrova K, Traykova-Andonova M, Mask A, Haldane D, Hatchette TF. High Seroprevalence of Jamestown Canyon Virus among Deer and Humans, Nova Scotia, Canada. Emerg Infect Dis 2018 Jan;24(1):118-21. DOl PubMed

49. Rocheleau JP, Michel P, Lindsay LR, Drebot M, Dibernardo A, Ogden NH, Fortin A, Arsenault J. Emerging arboviruses in Quebec, Canada: assessing public health risk by serology 
in humans, horses and pet dogs. Epidemiol Infect 2017 Oct;145(14):2940-8. DOl PubMed

50. Sampasa-Kanyinga $H$, Lévesque $B$, Anassour-Laouan-Sidi E, Côté S, Serhir B, Ward BJ, Libman MD, Drebot MA, Makowski K, Dimitrova K, Ndao M, Dewailly E. Zoonotic infections in communities of the James Bay Cree territory: an overview of seroprevalence. Can J Infect Dis Med Microbiol 2013;24(2):79-84. DOI PubMed

51. Agence de la santé publique du Canada. Prévention du virus du Nil occidental. Ottawa (ON) : ASPC; 2016. https://www. canada.ca/fr/sante-publique/services/maladies/virus-niloccidental/prevention-virus-nil-occidental.html

52. World Health Organization. A global brief on vector-borne diseases. Geneva (CH): WHO; 2014. www.who.int/ campaigns/world-health-day/2014/global-brief/en/
53. Uehlinger FD, Wilkins W, Godson DL, Drebot MA. Seroprevalence of Cache Valley virus and related viruses in sheep and other livestock from Saskatchewan, Canada. Can Vet J 2018 Apr;59(4):413-8. PubMed

54. Brown HE, Young A, Lega J, Andreadis TG, Schurich J, Comrie A. Projection of Climate Change Influences on U.S. West Nile Virus Vectors. Earth Interact 2015 Dec;19(18):18. DOI PubMed

55. Faust CL, McCallum HI, Bloomfield LS, Gottdenker NL, Gillespie TR, Torney CJ, Dobson AP, Plowright RK. Pathogen spillover during land conversion. Ecol Lett 2018 Apr;21(4):471-83. DOl PubMed

56. Iranpour M, Turell MJ, Lindsay LR. Potential for Canadian mosquitoes to transmit Rift Valley fever virus. J Am Mosq Control Assoc 2011 Dec;27(4):363-9. DOI PubMed 